\title{
STABILITY OF ANTI-ACNE NIOSOME GELS CONTAINING BETEL LEAF (PIPER BETLE L.) ESSENTIAL OIL
}

\author{
MAHDI JUFRI ${ }^{1 *}$, MUTHAHARRAH MUTHAHARRAH ${ }^{1}$, ELSA HUMAIRAH ${ }^{1}$, ERNIE HERMAWATI PURWANINGSIH ${ }^{2}$
}

${ }^{1}$ Department of Pharmaceutical Science, Faculty of Pharmacy, Universitas Indonesia, Depok, Indonesia. ${ }^{2}$ Department of Pharmacy, Faculty of Medicine, Universitas Indonesia, Depok, Indonesia. Email: mahdi.jufri@farmasi.ui.ac.id

Received: 21st April 2017, Revised and Accepted: 13th July 2017

\section{ABSTRACT}

Objective: Formulation, antibacterial activity, and stability tests of niosomal gels containing betel leaf (Piper betle L.) essential oil as an anti-acne treatment were carried out. Niosome vesicular carriers provide drug delivery through the topical and transdermal routes. The aim of creating the niosome preparation was to increase the transfollicular penetration and improve the stability of the gel.

Materials and Methods: Betel leaf essential oil extraction was performed using the steam distillation method, and essential oil compound identification was completed using gas chromatography-mass spectrometry. The niosome formulations were generated with two cholesterolsurfactant amount ratios, specifically, 1:1 (F1) and 1:2 (F2; w/w). The niosomes were evaluated, including the entrapment efficiency test, using ultraviolet-visible spectrophotometry; particle size analysis was performed using a particle size analyzer; and the vesicle morphology test was conducted using transmission electron microscopy. The niosomes were made into a gel using $0.5 \%$ carbopol 940 as the gelling agent. The niosome gels were evaluated for their organoleptic properties, $\mathrm{pH}$, viscosity, antibacterial activity against Propionibacterium acnes, and stability for 12 weeks at three different storage temperatures, namely, low temperature $\left(4 \pm 2^{\circ} \mathrm{C}\right)$, room temperature $\left(28 \pm 2^{\circ} \mathrm{C}\right)$, and high temperature $\left(40 \pm 2^{\circ} \mathrm{C}\right)$.

Results: The test results showed that the F2 niosome gel was more stable than the F1 gel was, while the antibacterial activities of the F1 and F2 niosome gels did not differ significantly.

Conclusion: The niosomal gel preparations' inhibition of the growth of P. acnes bacteria was decreased compared with that of the essential oils

Keywords: Gas chromatography-mass spectrometry, Niosomes, Propionibacterium acnes, Particle size analysis, Transmission electron microscopy.

(C) 2017 The Authors. Published by Innovare Academic Sciences Pvt Ltd. This is an open access article under the CC BY license (http://creativecommons. org/licenses/by/4. 0/) DOI: http://dx.doi.org/10.22159/ijap.2017.v9s1.72_79

\section{INTRODUCTION}

There is a high prevalence of acne in Indonesia; among females, the rates of acne by ethnicity are $37 \%$ for African Americans, 32\% for Hispanics, 30\% for Asians, 24\% for Caucasians, and 23\% for Indians [1]. Various anti-acne drugs, particularly those made of herbal ingredients, have been developed to improve the therapy. Indonesia has many native plants that are effective when it comes to overcoming acne problems; one of these is the betel leaf, which contains cavichol, a substance with a potent bactericidal effect, which is around 5 times stronger than that of ordinary phenol [2].

In general, topical preparations have three lines of penetration; among other, they have intracellular, transcellular, and transfollicular pathways. Of these three lines, the transfollicular path is the most overlooked, as hair follicles constitute only $0.1 \%$ of the total surface area of the skin. Nevertheless, the penetration and absorption of compounds through this pathway can be much deeper under the skin than seen along the transdermal route. Therefore, the path of drug delivery through the hair follicles has a great potential in the treatment through the topical route.

Drug delivery systems based on nanotechnology are constantly being developed to track the transfollicular delivery of niosomes. Niosome vesicular carriers provide drug delivery through the topical and transdermal routes. Such carriers can carry hydrophobic and amphiphilic drug molecules [3]. Niosomes can improve the skin penetration and increase drug accumulation in the superficial skin stratum, while the systemic absorption of the drug into the pathway can be reduced [4].

Niosomes have the same functions as liposomes. They can enhance the therapeutic index of a drug and carry drugs directly to the target cells.
However, niosomes have several advantages compared to liposomes, as follows: Lower toxicity, relatively low cost, and greater stability, meaning that niosomes do not require special storage conditions [5]. Thus, niosome can be used as a drug delivery system to treat skin disorders. When applied topically, niosomes can increase the residence time of the drug in the stratum corneum. They can also change the properties of the stratum corneum by reducing the loss of water in transepidermal lines and improving skin smoothness by reducing the number of skin lipids lost. Furthermore, the manufacturing of niosomes is relatively inexpensive compared to that of liposomes.

The potential of essential oils in plants has been widely studied, especially in terms of alternative treatments, such as treatment for various infectious diseases, alternative pharmaceutical medicines, and natural therapies [6]. The betel leaf contains tannins, sugar, vitamins, and essential oils. Organoleptic betel oil is a yellow or brownish liquid with a bitter taste it is soluble in organic solvents, such as alcohol, ether, and chloroform, while it is water insoluble [7]. The percent of volatile oil content in betel leaves ranges from $0.7 \%$ to $2.6 \%$. Approximately $60-80 \%$ of the leaves are made up of phenylpropanes, such as eugenol $(42.5 \%)$, estragole $(14.6 \%)$, and carvacrol $(4.8 \%)$. The active compounds exhibit antibacterial and antifungal efficacy.

In one study, only $0.1 \%$ of colonies of Streptococcus sp. bacteria could survive after the administration of $0.5 \%$ betel leaf essential oils [7]. One ingredient of essential oil exhibiting antibacterial properties is cavichol. The bacterial killing power of cavichol compound is 5 times greater than that of ordinary phenol compounds. Cavichol also gives betel its distinctive, sharp odor. The compounds responsible for the unpleasant aroma and antiseptic properties are phenol compound. Given these considerations, the objectives of this study were to determine the 
antibacterial effectiveness of betel leaf (Piper betle L.) essential oils against Propionibacterium acnes in comparison with clindamycin and determine the stability of a cavichol niosome-containing gel formula containing betel leaf $(P$. betle $\mathrm{L}$.) essential oils.

\section{MATERIALS AND METHODS}

\section{Material}

Betel leaves $(P$. betle L.) were obtained the Herbarium Bogoriense Lembaga Ilmu Pengetahuan Indonesia Cibinong, Bogor. For the bacterial test and media, standard germ P. acnes (ATCC 11822) was obtained (Microbiology Laboratory Medicine), and Müller-Hinton agar (MHA) powder medium (Oxoid) was used.

\section{Material formulations and other materials}

Sorbitan monostearate (Kosteran ${ }^{\circledR}$ ), cholesterol (Sigma), Carbomer 940 (Merck), gallic acid standards (Sigma), Folin-Ciocalteu's phenol reagent, phosphate buffer ( $\mathrm{pH}$ 7.4), $0.9 \% \mathrm{NaCl}$, and McFarland III solution were used. Furthermore, 6-mm paper disks, $0.22-\mu \mathrm{m}$ membrane bacteria filters, and clindamycin $(2 \mathrm{~g}$ ) antibiotic in a size of $6 \mathrm{~mm}$ paper disks (Oxoid) were used in the experiment. Finally, steam distillation of water, a rotary evaporator (Hahn Shin), particle size analyzer (PSA), gas chromatograph-mass spectrometry (GC-MS) equipment, transmission electron microscope (JEOL JEM-1400), ultraviolet-visible (UV-Vis) spectrophotometer (Shimadzu UV-1800), centrifuge (Kubota 5100), climatic chamber (Memmert), pH meter (Corning), Brookfield viscometer, and incubator were employed.

\section{Method}

Before distillation, $10 \mathrm{~kg}$ of betel leaves were withered to obtain a sample weight of $1 \mathrm{~kg}$. The distillation method used was steam distillation. Once distilled, $\mathrm{Na}_{2} \mathrm{SO}_{4}$ was added to absorb the water in the oil ( $2 \%$ of the volume of oil or to taste) to obtain pure oil.

\section{Analysis of components of betel oil compounds}

Testing was carried out using Agilent Technologies 6890N GC-MS instruments. The amounts of the compounds contained in the essential oil of betel leaf were indicated by the numbers that appearing on the chromatogram peaks.

\section{The minimum inhibitory concentration (MIC) test betel leaf volatile oil}

The medium used was MHA. Swabs of $P$. acnes bacterial cultures that were still fresh and these were diluted in $2 \mathrm{ml}$ physiological saline solution until turbidity according to the McFarland III solution (109 bacteria/ml). Then, the inoculum solution was scratched evenly into MHA medium using sterilized cotton buds (swabs). Sample solutions are with concentrations of $0.25 \%, 0.5 \%, 1 \%, 2 \%, 4 \%$, and $8 \% \mathrm{v} / \mathrm{v}$ were created in n-hexane solvent. Furthermore, up to $20 \mathrm{ml}$ of sterile test solution was dropped on paper discs and then incubated for $24-48 \mathrm{hrs}$ at $37^{\circ} \mathrm{C}$ in an anaerobic jar. The same test was also conducted on the positive control clindamycin gel $(2 \mathrm{~g})$. Composition of material for niosome production and for production of niosomal gel $(20 \mathrm{~g})$ is available in Tables 1 and 2, respectively.

Niosomes were made using a thin-layer hydration method. Span 60 , cholesterol, and essential oil were dissolved in $10 \mathrm{ml}$ of dichloromethane (DCM) and stirred gently until all components were dissolved. DCM was removed using a rotary evaporator with a temperature of $41 \pm 2^{\circ} \mathrm{C}$ at a speed of 100 rpm under a vacuum for 50-60 minutes until no DCM was present. Furthermore, the niosomes were hydrated by adding $10 \mathrm{ml}$ of phosphate buffer.

The entrapment efficiency method was carried out to test total phenolics following Singleton and Rossi (1965) and using Folin-Ciocalteu's reagent. Tests were conducted on the total phenolic essential oil and the supernatant suspension niosomes. The supernatant was measured by UV-Vis spectrophotometry at a wavelength of $760 \mathrm{~nm}$. Phenol levels
Table 1: Composition of material for niosome production

\begin{tabular}{lll}
\hline Material & Weight & \\
\cline { 2 - 3 } & F1 & F2 \\
\hline DCM & $10 \mathrm{ml}$ & $10 \mathrm{ml}$ \\
Volatile oil & $200 \mathrm{mg}$ & $200 \mathrm{mg}$ \\
Span 60 & $100 \mathrm{mg}$ & $100 \mathrm{mg}$ \\
Cholesterol & $100 \mathrm{mg}$ & $100 \mathrm{mg}$ \\
Phosphate buffer & $10 \mathrm{ml}$ & $10 \mathrm{ml}$ \\
\hline DCM: Dichloromethane & &
\end{tabular}

Table 2: Composition of material for production of niosomal gel (20 g)

\begin{tabular}{lll}
\hline Material & Weight & \\
\cline { 2 - 3 } & F1 & F2 \\
\hline Niosome suspension & $10 \mathrm{~g}$ & $10 \mathrm{~g}$ \\
Carbomer 940 & $100 \mathrm{mg}$ & $100 \mathrm{mg}$ \\
TEA & $0.08 \mathrm{ml}$ & $0.08 \mathrm{ml}$ \\
Demineralized water & Aliquoted to $20 \mathrm{~g}$ & Aliquoted $20 \mathrm{~g}$ \\
\hline
\end{tabular}

TEA: Triethanolamine

Table 3: Results of the calculation of the yield of $P$. betle leaf essential oil

\begin{tabular}{llll}
\hline Distillation & Sample weight (g) & $\begin{array}{l}\text { Volume of } \\
\text { volatile oil (ml) }\end{array}$ & Yield (\%) \\
\hline 1 & 1.000 & 6 & 0.6 \\
2 & 1.000 & 11 & 1.1 \\
\hline
\end{tabular}

P. betle: Piper betle

sequestered in niosomes were calculated using the following formula: EJ $(\%)=($ Total phenol oil betel-total phenol supernatant niosome $) /$ (Total phenol oil betel) $\times 100 \%$.

The particle size distribution of the average diameter of niosome vesicles F1 and F2 are determined using a PSA. The morphological measurement of the vesicles was carried out using JEOL JEM-1400 transmission electron microscopy (TEM) instruments; then, the morphological structure of the vesicles was observed at various magnifications. Viscosity tests were carried out using a Brookfield viscometer with spindle number 4. Meanwhile, to determine antibacterial activity of the niosomal gel preparation, the same MIC for testing betel oil was established using the agar diffusion method. The medium used was MHA. Temperature stability tests were carried out at three different storage temperatures, namely, low temperature $\left(4 \pm 2^{\circ} \mathrm{C}\right)$, room temperature $\left(28 \pm 2^{\circ} \mathrm{C}\right)$, and high temperature $\left(40 \pm 2^{\circ} \mathrm{C}\right)$. In addition, the $\mathrm{pH}$ of the preparation was determined. These observations were performed every 2 weeks for 12 weeks.

For the cycling test, both the F1 and F2 formulations were stored at low temperature $\left(4 \pm 2^{\circ} \mathrm{C}\right)$ for $24 \mathrm{hrs}$. Then, the preparation was transferred to a high temperature $\left(40 \pm 2^{\circ} \mathrm{C}\right)$ for $24 \mathrm{hrs}$. The procedure was repeated 6 times.

\section{RESULTS}

The volumes of essential oil obtained after the distillation process were 6 and $11 \mathrm{ml}$, respectively. From these data, the essential oil yield could be calculated using the following formula: \% Yield $=$ (Volume of essential oil)/(weight of crude drug) $\times 100 \%$. (Table 3)

Essential oils were first selected for analysis using GC-MS instruments they exhibited a clear yellow oil appearance. The analysis results were obtained in the form of a chromatogram (Fig. 1). 
Table 4: Analysis of volatile components in P. betle leaf oil using GC-MS

\begin{tabular}{llll}
\hline Peak & Retention time (minutes) & Area (\%) & Compound \\
\hline 1 & 5.743 & 6.1091 & Sabinen \\
2 & 8.733 & 14.8239 & Cavichol \\
3 & 10.097 & 31.0115 & Cavichol acetate \\
4 & 10.476 & 21.5984 & 4 -cyclopropyl-2-methoxy phenol \\
5 & 11.192 & 5.7105 & Caryophyllene \\
6 & 11.767 & 5.5838 & $\gamma$-murolene \\
7 & 12.202 & 9.1973 & 3 -allyl-6-methoxy phenyl acetate \\
8 & 13.553 & 5.9655 & Allylpyrocatechol diacetate \\
\hline
\end{tabular}

GC-MS: Gas chromatograph-mass spectrometry, P. betle: Piper betle

\section{MIC testing of betel leaf volatile oil}

The test results showed a clear zone around the paper disk and no bacterial growth. The greater the concentration of essential oils, the greater the inhibition zone that was produced. Inhibitory zone diameters were measured using calipers, and betel oil showed an MIC of $0.5 \%$ with an inhibition diameter of $9.10 \mathrm{~mm}$ (Table 4). The inhibition test was also performed on the positive control, clindamycin gel $(2 \mathrm{~g})$. Compared with essential oils, the antibacterial activity of clindamycin was much stronger; with diameters of inhibition of $58.6 \mathrm{~mm}$. Results of MIC from betel leaf volatile oil and niosomes' entrapment efficiency are described in Tables 5 and 6, respectively.

\section{Particle size distribution}

Measurement of particle size distribution was carried out for both niosome formulations, F1 and F2, using the PSA instrument (Table 7).

\section{Vesicle morphology}

The niosome formulation used in the vesicle morphology test was F1, as the previous tests showed that its particle size distribution and entrapment efficiency are superior to those of F2. The test results on the vesicle morphology were determined using TEM images taken from two different angles, as shown in Fig. 2. A grain-like vesicle enclosing the oil droplets was apparent from a magnification of $\times 10,000$ (Fig. 2b). The vesicle-like structure was thought to represent niosome lamellar. The lamellar suspension niosome was single lamellar vesicles, and multilamellar vesicles were observed.

\section{The antibacterial activity test}

Niosome antibacterial gel testing was performed using the paper disc diffusion method, including MIC testing of betel leaf essential oil. The results showed a clear zone around the paper disk (Table 8).

\section{The temperature stability test}

During storage at low temperatures, the preparation remains in gel form, and there is no phase separation in storage for 12 weeks. At room temperature, formulations F1 and F2 were changed in color after storage for 4 weeks. During storage at high temperatures, there was significant discoloration in preparations of F1 and F2. The F1 preparation showed a brownish color, but this formulation was more concentrated than F2 was. In Figs. 3 and 4 both F1 and F2 tended to show $\mathrm{pH}$ fluctuation during storage at the three different temperatures.

\section{DISCUSSION}

From the analysis of compounds of betel leaf essential oils using GC-MS, it was concluded that most of the expected compounds were phenols, including cavichol, cavichol acetate, 4-cyclopropyl-2-methoxyphenol, 3-allyl-6-methoxy phenyl acetate, and allylpyrocatechol diacetate. There were also compounds from the classes of sabinene monoterpenes and sesquiterpenes, namely, caryophyllene and $\gamma$-murolene. The similarity of these compounds to the comparator compounds approached $100 \%$. The compound with the greatest concentration was cavichol acetate, with an area of $31.01 \%$. Essential oils' activity in inhibiting the growth of bacteria is caused by the content of phenolic compounds, one of which — cavichol-is efficacious as a bactericide, defined as a substance that can kill vegetative forms of bacteria.
Table 5: Results of MIC from betel leaf volatile oil

\begin{tabular}{ll}
\hline Concentration (\%) & Diameter zone of MIC (mm) \\
\hline 8 & 12.45 \\
4 & 11.00 \\
2 & 9.50 \\
1 & 9.10 \\
0.5 & 9.10 \\
0.25 & No MIC \\
\hline
\end{tabular}

MIC: Minimum inhibitory concentration

Table 6: Niosomes' entrapment efficiency

\begin{tabular}{ll}
\hline Formula & EJ (\%) \\
\hline F1 & 88.99 \\
F2 & 88.74 \\
\hline
\end{tabular}

Table 7: Results for niosome particle size

\begin{tabular}{lll}
\hline \multirow{2}{*}{ Parameter } & \multicolumn{2}{l}{ Result (nm) } \\
\cline { 2 - 3 } & F1 & F2 \\
\hline$Z_{\text {average }}$ & 254.96 & 856.23 \\
Dv10 & 169.87 & 489.91 \\
Dv50 & 295.20 & 933.50 \\
Dv90 & 513.00 & 1862.58 \\
PDI & 0.2130 & 0.2480 \\
\hline
\end{tabular}

PDI: polydispersity index

Table 8: Diameter of the MIC zone of P. acnes

\begin{tabular}{ll}
\hline Formula & Diameter $(\mathbf{m m})$ \\
\hline F1 & 7.70 \\
F2 & 7.20 \\
\hline
\end{tabular}

MIC: Minimum inhibitory concentration, P. acnes: Proprionibacterium acnes

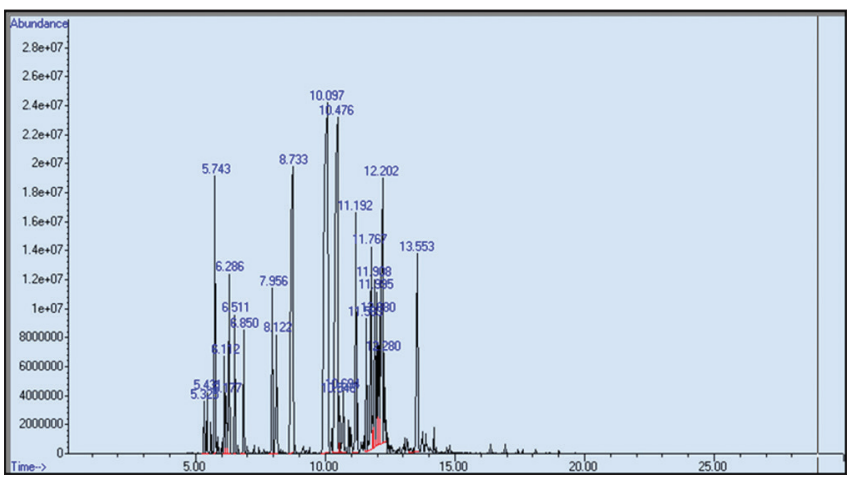

Fig. 1: Chromatogram of betel leaf essential oil components using gas chromatography-mass spectrometry instruments 


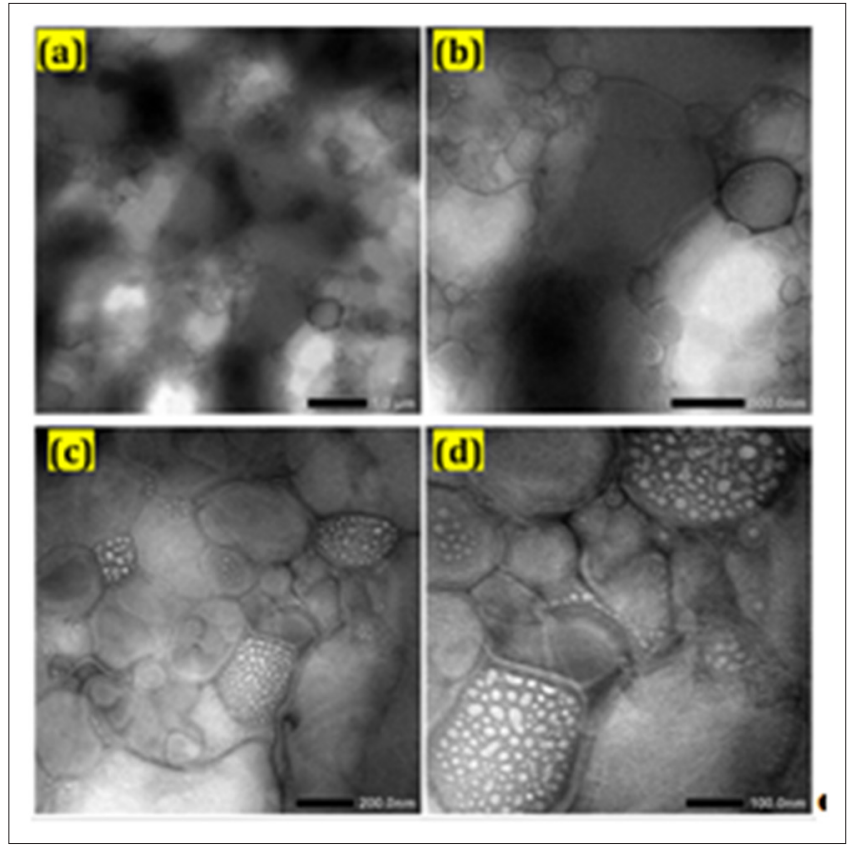

Fig. 2: Morphology niosome F1 magnification (a) 5000, (b) 10,000 (c) 20,000 (d) 40,000 times

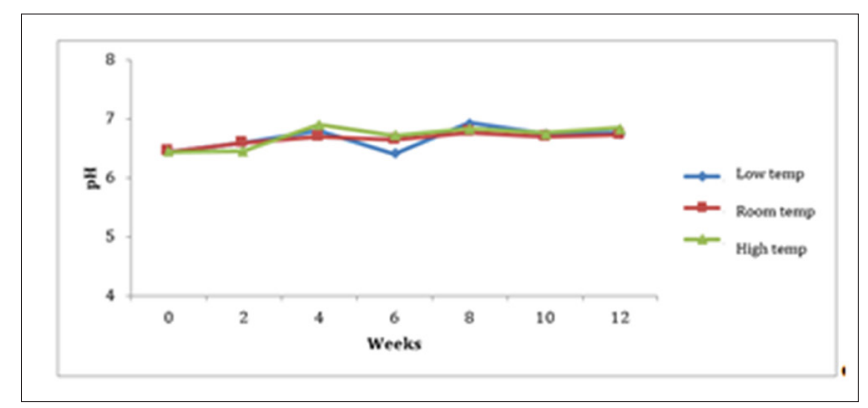

Fig. 3: $\mathrm{pH}$ values of the F1 niosome gel over 12 weeks

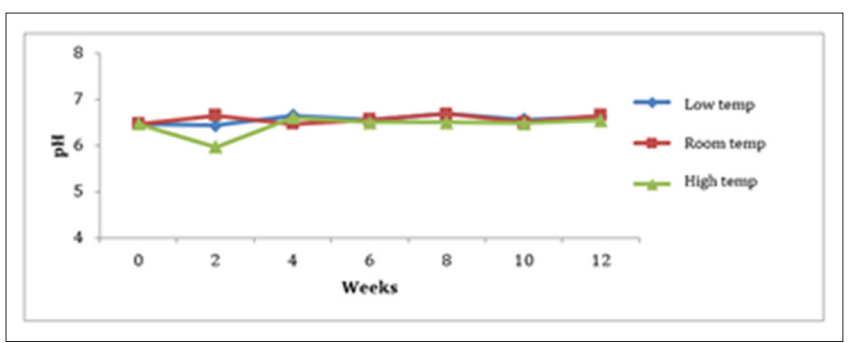

Fig. 4: pH values of the F2 niosome gel over 12 weeks

In this study, the selection of span 60 as a carrier vesicle was also influenced by the value of its phase transition temperature, which is higher $\left(50^{\circ} \mathrm{C}\right)$ than the temperatures of other surfactants. The use of a surfactant can increase the entrapment efficiency of niosomes. In addition, span 60 has better stability compared with other surfactants [8]. Niosome products were also generated using cholesterol to form stiffness and prevent leakage of the vesicles so that the rows of lipid molecules could be packed in the lipid bilayers of niosome vesicles. The niosome products were generated using the thin-layer hydration method. Direct vesicles were formed when niosome hydrated; these were characterized by the formation of a white suspension. The hydration process aims to form vesicles and improve the entrapment efficiency of niosomes.
The entrapment efficiency of niosomes is highly dependent on the stability of the vesicles, which also depends on the type and amount of surfactant bilayer, cholesterol, and drug [9]. Theoretically, the more span 60 were added, the greater the entrapment efficiency generated. However, the results showed that F1 had greater efficiency than F2, although the difference was not statistically significant. Presumably, this was influenced by the addition of the same amount of cholesterol, which can stabilize many bilayer membranes. The particle size distribution differences were significant because the number of additions of span 60 was different in the two formulations. Certain surfactants, including span 60, can form large niosome vesicles. The more span 60 is added to the formula, the larger the niosome vesicles will be. Nevertheless, it led to span 60 has entrapment efficiency the percentage increased than other surfactants as well [10].

In addition to the type and amount of surfactant, the size of niosome particles is also influenced by the type of material that is sequestered, in this case, essential oils. The interaction of oil with the head of the surfactants resulted in bilayer repulsion due to the increased load; this resulted in increased particle sizes [11]. Moreover, several other factors may have affected the particle size of F2 compared with F1; for instance, aggregation was carried out before testing due to the length of time between manufacturing and the measurement of particle size distribution. Niosome gel antibacterial potency testing was carried out in relation to the growth of $P$. acnes. When compared with the antibacterial activity of the betel leaf oil, it is reflecting the decline in the ability of the active substance to kill the bacteria $P$. acnes when added to the niosomal gel preparation. The inhibition capacity of $2 \%$ betel leaf oil was $9.5 \mathrm{~mm}$, whereas those of the niosomal gels, F1 and F2, were 7.70 and $7.20 \mathrm{~mm}$, respectively. This means that a strong decline in antibacterial activity occurred. This may be because volatile oil was entrapped in vesicles, resulting in suboptimal volatile oil release due to being blocked by the vesicle membrane. These results are comparable with those of previous research, which examined the antibacterial activity of gallidermin niosomes compared with that of gallidermin without niosomes [12]. The results also showed a decrease in gallidermin's antibacterial activity against $P$. acnes and Streptococcus aureus. Nevertheless, niosomal gel is still considered to increase the penetration of essential oils into the skin. In testing the stability according to temperature (Figs. 3 and 4), it was found that low-temperature storage can reduce the oxidation of volatile oil under heat, allowing the preparations to remain relatively stable.

\section{CONCLUSION}

Niosomes containing essential oils could be made with betel leaves and span 60 with cholesterol ratios of 1:1 and 1:2. The niosomal gel preparations' inhibition of the growth of P. acnes bacteria was decreased compared with that of the essential oils that were not included in a formulation. The niosomal gel preparation was more stable under low-temperature storage than at room temperature, and it was most unstable when stored at a high temperature. Differences between the span of 60 formulations of niosomes affected the stability of the preparations. Comparison of cholesterol and surfactant ratios showed that a ratio of $1: 2(\mathrm{w} / \mathrm{w})$ was more stable than a $1: 1(\mathrm{w} / \mathrm{w})$ ratio.

\section{REFERENCES}

1. Perkins AC, Cheng CE, Hillebrand GG, Miyamoto K, Kimball AB. Comparison of the epidemiology of acne vulgaris among Caucasian, Asian, Continental Indian and African American women. J Eur Acad Dermatol Venereol 2011;25(9):1054-60.

2. Heyne K. Tumbuhan Berguna Indonesia. Jakarta: Badan Litbang Departemen Kehutanan; 1987.

3. Madhav NV, Saini A. Niosomes: A novel drug delivery system. Int J Pharm Res Chem 2011;1(3):498-511.

4. Manconi M, Sinico C, Valenti D, Lai F, Fadda AM. Niosomes as carriers for tretinoin. III. A study into the in vitro cutaneous delivery of vesicle-incorporated tretinoin. Int J Pharm 2006;311(1-2):11-9.

5. Akhilesh D, Bini KB, Kamath JV. Review on span-60 based non-ionic 
surfactant vesicles (niosomes) as novel drug delivery. Int J Res Pharm Biomed Sci 2011;3(1):6-12.

6. Prabuseenivasan S, Jayakumar M, Ignacimuthu S. In vitro antibacterial activity of some plant essential oils. BMC Complement Altern Med 2006;6:39.

7. Rathee JS, Patro BS, Mula S, Gamre S, Chattopadhyay S. Antioxidant activity of piper betel leaf extract and its constituents. J Agric Food Chem 2006;54(24):9046-54.

8. Biswal S, Murthy PN, Sahu J, Sahoo P, Amir F. Vesicles of non-ionic surfactants (niosomes) and drug Delivery potential. Int J Pharm Sci Nanotechnol 2009;1(1):1-8.
9. Kumar GP, Rajeshwarrao P. Nonionic surfactant vesicular systems for effective drug delivery - An overview. Acta Pharm Sin B 2011;1(4):208-19.

10. Bhandari N. Review article: Proniosomes - A surrogate for transdermal drug delivery. Int J Pharm Res Biol Sci 2012;1(6):10-26.

11. Agarwal S, Bakshi V, Raghuram PV, Pandey S, Udupa N. Effect of cholesterol content and surfactant HLB on vesicle properties of niosomes. Indian J Pharm Sci 2004;1:121-3.

12. Manosroi A, Sritapunya T, Jainonthee P, Manosroi J, Anti P. Acne activity of cream containing aromatic volatile oil from Thai medicinal plants entrapped in niosomes for acne treatment. Thailand 2010;392:304-10. 